\title{
The Galactic Pulsar Population
}

\author{
Duncan R. Lorimer \\ Department of Physics, 210 Hodges Hall, Morgantown, WV 26506, USA \\ email: duncan.lorimer@mail.wvu.edu
}

\begin{abstract}
Simulations of the Galactic pulsar population are reviewed. These include snapshot and time-dependent models of normal pulsars as well as binary and milli-second pulsars.
\end{abstract}

The radio pulsar population currently known totals over 1650 objects in our Galaxy, thanks largely to a number of large-scale surveys carried out with the Parkes radio telescope, as well as Green Bank, Arecibo and the Giant Metrewave telescope (see Lorimer 2008). Due to the complexities in describing the population and related selection effects analytically, the main approach to the problem is through a Monte Carlo simulation of the Galactic distribution of pulsars, propagation effects in the interstellar medium and the various large scale surveys that have been carried out. Two main types of simulations are typically used: (i) a snapshot model of the population at the current epoch; (ii) a time dependent model following the spin and kinematic evolution over time. The former approach requires fewer assumptions and is easy to optimize. The latter approach is ultimately more reliable, but has more model dependent results and covariances between various parameters. With these caveats in mind, Lorimer et al. (2006) have produced a snapshot model of the Galactic population of normal (i.e. non-millisecond) pulsars and find that their radial distribution closely follows the assumed distribution of free electrons and has a scale height around 330 pc. Faucher-Giguère \& Kaspi (2006) have published a detailed time dependent model and find evidence for a log-normal luminosity function in which the luminosity depends upon spin parameters. They found that the birth velocity distribution is most likely distributed exponentially in each of the $x, y$ and $z$ components, with a mean three-dimensional speed of $380 \mathrm{~km} \mathrm{~s}^{-1}$. Models of the millisecond and binary pulsar population are less well established, but it is now clear that they form a dynamically relaxed population with a smaller velocity dispersion and a larger scale height of 500 pc. A recent study by Story et al. (2007) reproduces many of the key features of the observable millisecond pulsar population. Kiel (2009) has made significant progress in the development of a self-consistent model of the millisecond and normal pulsar population which includes the effects of binary evolution. Models are available at psrpop.phys.wvu.edu and psrpop.sourceforge.net.

\section{Acknowledgements}

I thank the AAS for travel support. My research is supported by the National Science Foundation, the Research Corporation and West Virginia EPSCoR.

\section{References}

Faucher-Giguère, C. A. \& Kaspi, V. M. 2006, ApJ, 643, 332

Kiel, P. 2009, PhD thesis, Swinburne University of Technology

Lorimer, D. R. et al. 2006, MNRAS, 372, 777

Lorimer, D. R. 2008, Living Rev. Relativity, 11

Story, S. R. et al. 2007, ApJ, 671, 713 